\title{
Editorial
}

\section{Monitoring and Modeling Terrestrial Ecosystems' Response to Climate Change 2016}

\author{
Dong Jiang, ${ }^{1}$ Gang Liu, ${ }^{2}$ and Yongping $\mathrm{Wei}^{3}$ \\ ${ }^{1}$ Key Laboratory for Resources Use and Environmental Remediation, Institute of Geographical Sciences \\ and Natural Resources Research, Chinese Academy of Sciences, Beijing, China \\ ${ }^{2}$ University of Southern Denmark, Odense, Denmark \\ ${ }^{3}$ University of Queensland, Brisbane, QLD, Australia
}

Correspondence should be addressed to Dong Jiang; jiangd@igsnrr.ac.cn

Received 23 October 2016; Accepted 23 October 2016

Copyright (C) 2016 Dong Jiang et al. This is an open access article distributed under the Creative Commons Attribution License, which permits unrestricted use, distribution, and reproduction in any medium, provided the original work is properly cited.

Nowadays great attention has been paid to the impact of global climate change on the terrestrial ecosystem and their adaption as well. Dataset and in-deep insights on the spatial and temporal dynamics of ecosystems' response to climate change are of great significance for global change related studies. This special issue, which consists of 6 articles, aims to present the typical recent advances in these areas.

Conservation services are key indices for measuring the terrestrial ecosystems' response to climate change. J. Zhai et al. provided a method for evaluating the water conservation service together with ecosystem conservation service based on multisource satellite imagery and in situ observation data. In their case study in the national key ecological function zones in China, spatial and temporal features of conservation services were analyzed from 2000 to 2014. Another useful index is the phenological calendar, which is a specialized calendar recording the sequence of phenological events. It is regarded as the fingerprints of climate change and has been widely used for assessing the impacts of climate change on the terrestrial ecosystems. F. Zheng et al. compiled the phenological calendars of 3 phenological observation stations in East China for the periods 1987-1996 and 2003-2012. It could be derived from those data that the beginning date of spring and summer in East China advanced, while the beginning date of autumn and winter delayed mainly because of the increasing of temperature. Another novel and interesting paper by Y. Liu et al. revealed that phenology could serve as an efficient methodology for monitoring and assessing the impact of climate change on plant-based tourist activities, such as fruit-picking.

Climate change has resulted in significant impacts on terrestrial water cycle. Z. Yang et al. analyzed the changes in evapotranspiration (ET) over the Loess Plateau in China based on the Global Land Data Assimilation System (GLDAS) data. It was proved that the Loess Plateau showed an increasing trend in potential ET compared to the decreasing trend in most other parts of the globe. Y. Zhou et al. proposed a regional water balance model based factor analysis method to identify the major factors that contribute to the variation of groundwater depth in Beijing Plain. The results indicated that the increasing domestic and environmental water use contributed greatly to the groundwater depletion.

Global change may lead to more frequent occurrence of meteorological hazards. B. Hou et al. tried to investigate whether earthquakes and rainstorm flood were correlated through a series of statistical analysis. The results revealed that some flood events might relate to earthquake events from a statistical perspective, which is especially true when the earthquake events happened in the vapor transmission zone where rainstorm events lead to abundant water vapors. In this regard, earthquake events are more likely to cause big rainstorm flood events.

This special issue presented the most recent findings and case studies in monitoring and modeling terrestrial ecosystems' response to climate change. 


\section{Acknowledgments}

We would like to thank all the authors and reviewers who contributed to this special issue.

$$
\begin{array}{r}
\text { Dong Jiang } \\
\text { Gang Liu } \\
\text { Yongping Wei }
\end{array}
$$



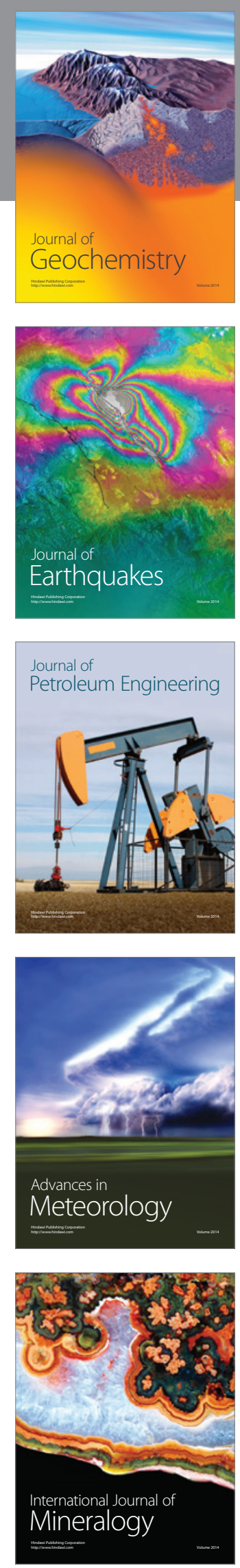
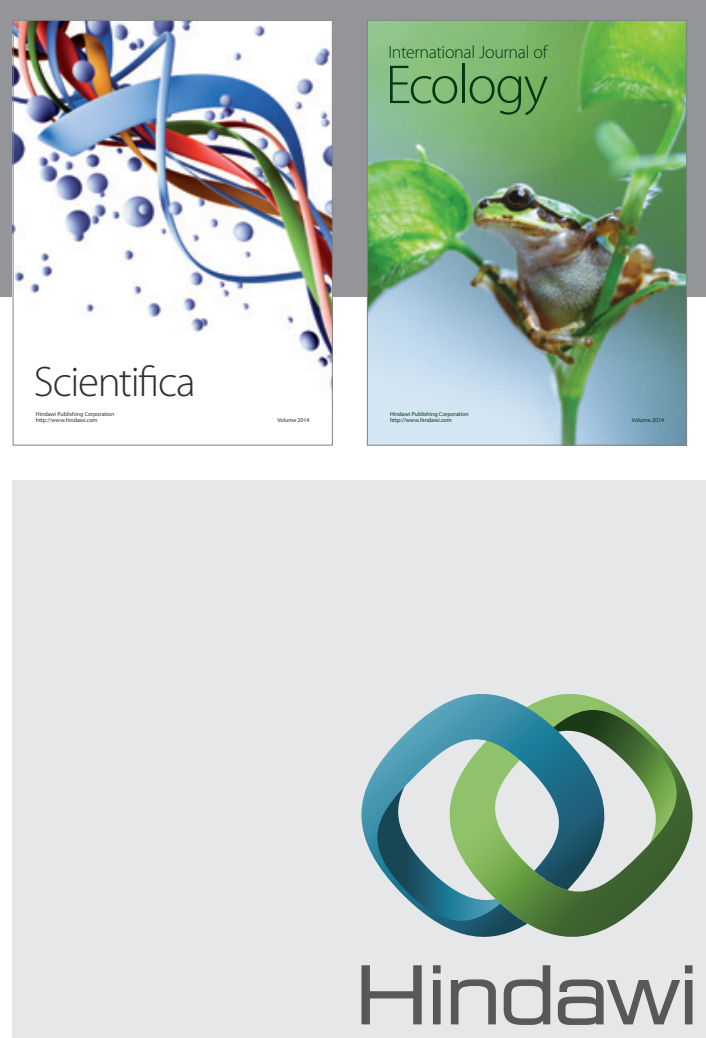

Submit your manuscripts at

http://www.hindawi.com
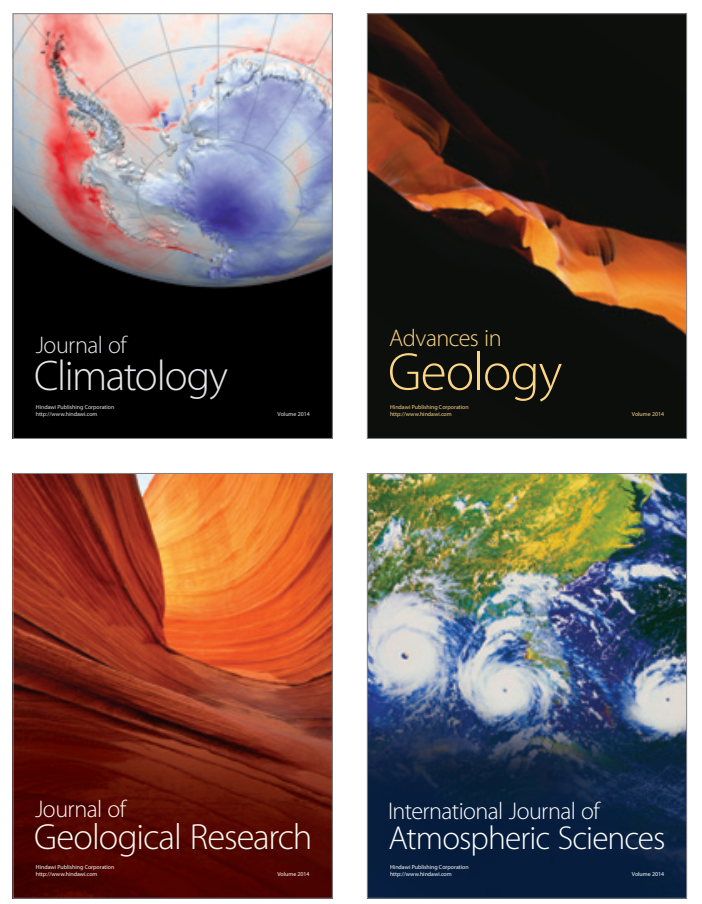

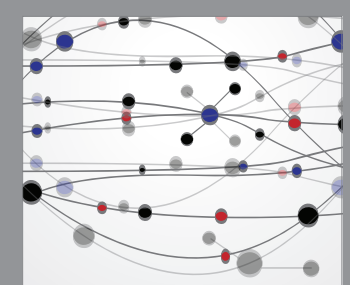

The Scientific

\section{World Journal}
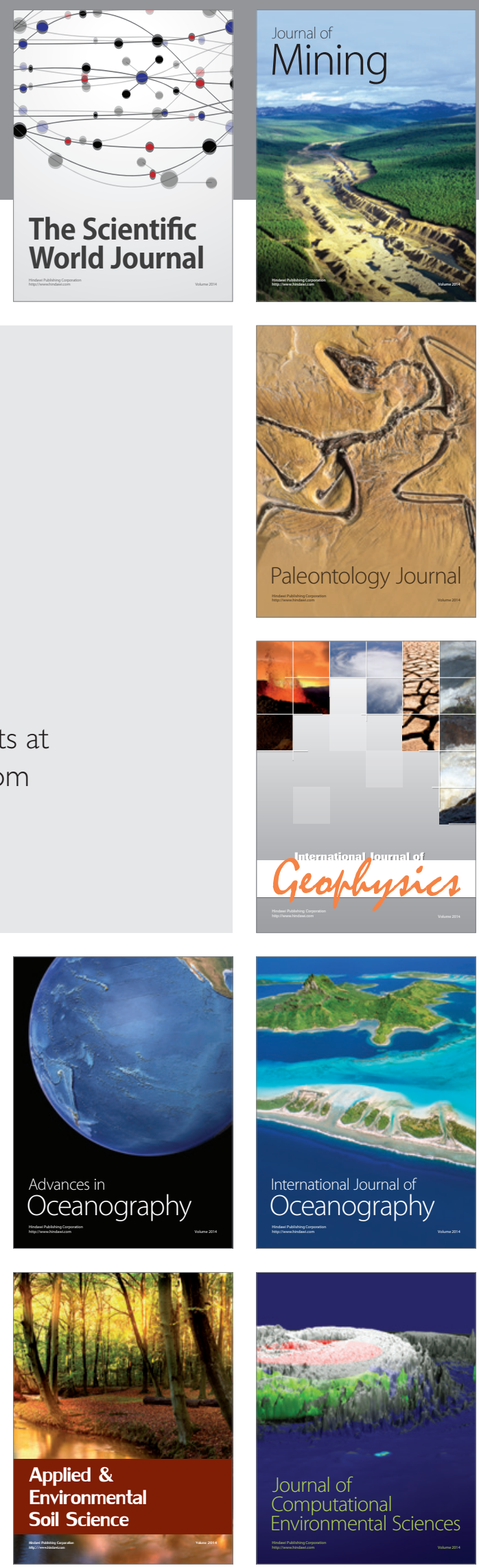